\title{
Proteins are New Molecular Markers When Analyzing Material Structures to Prevent Traumatic Brain Injuries
}

\author{
Hans von Holst ${ }^{1,2,3 *}$, Pasi Purhonen ${ }^{4}$, Daniel Lanner ${ }^{3}$, Ramakrishnan Balakrishnan Kumar ${ }^{4}$ and Hans Hebert ${ }^{4}$ \\ ${ }^{1}$ Section of Neurosurgery, Karolinska University Hospital, Sweden \\ ${ }^{2}$ Section of Research, Cenesy Company, Sweden \\ ${ }^{3}$ Section of Research, MIPS Company, Sweden \\ ${ }^{4}$ Department of Biosciences and Nutrition, Karolinska Institute and School of Engineering Sciences in Chemistry, Biotechnology and Health, KTH Royal \\ Institute of Technology, Huddinge, Sweden
}

*Corresponding author: Hans von Holst, Section of Neurosurgery, Karolinska University Hospital, 17176 Stockholm, Sweden, E-mail: hvh@cenesy.com

Received: 24 Jan, 2019 | Accepted: 08 Feb, 2019 | Published: 15 Feb, 2019

Citation: von Holst H, Purhonen P, Lanner D, Kumar RB, Hebert H (2019) Proteins are New Molecular Markers When Analyzing Material Structures to Prevent Traumatic Brain Injuries. J Neurol Neurobiol 5(1): dx.doi.org/10.16966/2379-7150.154

Copyright: (C) 2019 von Holst H, et al. This is an open-access article distributed under the terms of the Creative Commons Attribution License, which permits unrestricted use, distribution, and reproduction in any medium, provided the original author and source are credited.

\begin{abstract}
Development of new products for the prevention of traumatic brain injuries are mostly based on mechanical investigations. However, there is a demand for new and more sophisticated analyzes focusing on molecular levels to improve protective equipment. Recently we evaluated the mature protein laminin LN521 to find out the consequences to dynamic and semi-static impact and found substantial fragmentation and aggregation of the laminin structures. In the present study the effect of polymer materials used for reducing impacts were evaluated by using two different mechanical methods, denatured electrophoresis and electron microscopy. Thus, following dynamic impacts without and with the polymer materials the results showed a significant reduction of the force as well as the translational acceleration with up to over $50 \%$. Also, in the present laboratory investigation the mature laminin was used following dynamic impact to find out if this molecule can serve as a complementary analyzer to mechanical methods when searching for optimal protective materials. The results showed that the polymer materials had the capacity to save the laminin structures from both fragmentation and aggregation as evaluated with denatured electrophoresis and electron microscopy. Therefore, proteins may complement today's calculation simulations and mechanical investigations in the search for improved protective systems to the skull bone and brain tissue. The present result shows that laminin structures may become a valuable method to further identify new structures on a molecular level in the search for improved protective materials to the brain tissue at physical exercise and at accidents.
\end{abstract}

\section{Introduction}

The overall healthcare cost to governmental as well as nongovernmental authorities following injuries to the human body is substantial. The same holds true also in Sweden [1,2]. Although it is somewhat difficult to estimate the total sum on a global perspective it is concluded that the costs will increase enormously within the next decades. One of the reasons to that is the demographic scenario of a substantially increased elderly population. Today elderly people are very active in various sports. However, with growing age the human body does not keep in line with those activities. As a consequence, it can be foreseen that this will result in a significant increase in injuries such as brain and bone tissue among the young and adult population and especially among elderly people. Thus, the need for prevention of these injuries is huge. This demands the search for the development of new analyzing methods on a molecular level when it comes to evaluate different prevention products reducing traumatic brain injury and the consequences following both dynamic and static impacts. There is a number of materials alone or in various composite constructions having the capacity to reduce consequences following an accident. Of special interest is the prevention of mild, moderate and severe head injuries. So far most analyzing methods for this purpose are based on pure mechanical investigations and lately also on simulation methods. However, there is a need for more sophisticated laboratory investigations in the search for more advanced methods approaching substances on a molecular level in the human body. A potential analyzer for such purposes may be the protein laminin wide spread on a cellular level. In general, the laminin proteins are responsible for holding cells and tissues such as basement membranes together. Also, the laminins have both common and specific functions. One important common function is the interaction between receptors in plasma cell membranes close to the basement membranes. More specifically, the laminins may regulate some of the activities between cells and signaling pathways [3]. These 
metabolic functions may be jeopardized following traumatic brain injuries in all ages. Recently the mature protein structures of laminin were analyzed with electrophoresis and Electron Microscopy (EM) following dynamic and semi-static impacts. It was found that both impacts resulted in unfolding of the LN521 structures [4]. Thus, as a complement to existing simulation and mechanical methods laminin and other protein structures may be excellent structures to be used when analyzing the effects of composite materials for the prevention of especially traumatic brain injury.

The purpose of the present investigation was

to evaluate the consequences for laminin structures upon dynamic impact without and with polymer materials aiming at the development of new analysis methods on molecular levels in the search for effective products to prevent traumatic brain injury and dementia all ages.

\section{Materials and Methods}

\section{Mechanical investigation}

The first mechanical laboratory method for dynamic impact with subsequent analysis of the $774 \mathrm{kDa}$ laminin molecule was a dummy consisting of a steel body form of $5 \mathrm{~kg}$ weight falling from $60 \mathrm{~cm}$ height and at an impact speed of $3.3 \mathrm{~m} / \mathrm{s}$ or $11.9 \mathrm{~km} / \mathrm{h}$ (Figure 1a) [5]. The second method was a dummy consisting of a Hybrid III head form of $3.9 \mathrm{~kg}$ weight falling from $55 \mathrm{~cm}$ height and at an impact speed of 3.1 $\mathrm{m} / \mathrm{s}$ or $11.2 \mathrm{~km} / \mathrm{h}$ (Figure $1 \mathrm{~b}$ ) [6,7]. Normally, a number of different materials are analyzed in the search for the optimal material to reduce an impact force to the head. In the present analysis a Styrene Butyric Rubber was chosen as it has not been considered in this regard. Thus, for the dummy steel body form (Figure 1a) the investigated polymer structure analyses of laminin, in all 10, were defined as Styren Butyric Rubber (SBR) and a hardness of Sh 60 and Sh 81 with spikes (Figure 2a). For the dummy Hybrid III head form (Figure 1b) the investigated polymer structures, in all another 10 analyses, were defined as SBR and with a hardness of Sh50 and Sh70 (Figure 2b). The polymer structures were placed between the basal plate and the respective dummy. After impact the polymer structures were analyzed with regard to forces and translational acceleration and compared with that of control. The first laminin solution of $0.2 \mathrm{ml}$ were the control and analyzed without any impact. The second laminin solution of $0.2 \mathrm{ml}$, in all 10 solutions, was exposed to a dynamic impact by that shown in figure 1a. The third laminin solutions of $0.2 \mathrm{ml}$, in all 10 solutions, were exposed to a dynamic impact as shown in figure $1 \mathrm{~b}$. Following the dynamic impacts all laminin solutions were evaluated with denatured electrophoresis and $\mathrm{EM}$, respectively.

\section{Denatured electrophoresis}

The samples were analyzed by denaturing polyacrylamide gel electrophoresis (PAGE). In denaturing electrophoresis, both the treated and untreated laminin samples were mixed with $5 x$ loading buffer containing sodium dodecyl sulphate (SDS) and heated at $65^{\circ} \mathrm{C}$ for 10 minutes before loading on the $4-12 \%$ (steel body form) or $14 \%$ (Hybrid III head form). Tris-glycine gel (Thermo Fisher Scientific) [8]. The electrophoresis was then started by applying $150 \mathrm{~V}$ and ran until the blue front reaches the end of the gel [9]. The bands in the gel were visualized by silver staining using Silver quest silver ${ }^{\mathrm{TM}}$ staining kit (Thermo Fisher Scientific).

\section{Electron microscopy (EM)}

1-3 $\mu \mathrm{l}$ aliquots of the impacted specimen were applied to glowdischarged carbon-coated copper grids, blotted and stained $30 \mathrm{~s}$ with $2 \%(w / v)$ uranyl acetate $[10,11]$. The grids were checked using JEOL JEM-2100f transmission electron microscope (JEOL, Japan) operated at $200 \mathrm{kV}$. Images were collected with TVIPS TemCam-F415 $4 \mathrm{k} \times 4 \mathrm{k}$ CCD-camera (Tietz Video and Image Processing Systems GmbH, Gauting, Germany) using a nominal magnification of 80,000.

\section{Results}

\section{Dynamic impact with steel head form}

When evaluating the ten laminin solutions after the dynamic impact from the steel body (Figure 1a), two of them are represented in the present results. The force was reduced from $18.84 \mathrm{kN}$ in the reference wooden board (Figure 3) to $14.76 \mathrm{kN}$ or $22 \%$ with the polymer Sh 60 while the polymer Sh 81 reduced the force to $8.28 \mathrm{kN} 56 \%$. Also, compared to the reference wooden board with acceleration of $256.23 \mathrm{~g}$ it is shown that the polymer Sh 60 reduced the acceleration to 195.37 g or $24 \%$ reduction of the translational acceleration while the polymer Sh 81 reduced the translational acceleration to $118.11 \mathrm{~g}$ or $54 \%$. The impact speed did not differ between the control of Reference wooden board and the two polymer structures about $3.3 \mathrm{~m} / \mathrm{s}$ or $12 \mathrm{~km} / \mathrm{h}$.
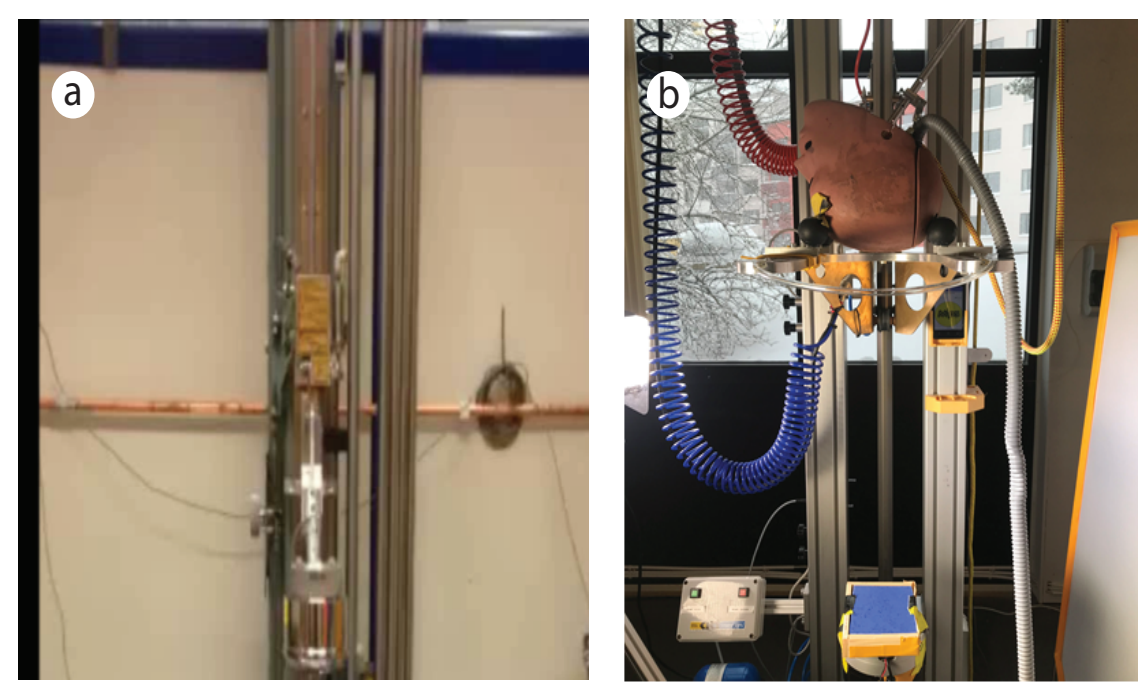

Figure 1: Showing the steel body form (1a, left) and Hybrid head form (1b, right).

Citation: von Holst H, Purhonen P, Lanner D, Kumar RB, Hebert H (2019) Proteins are New Molecular Markers When Analyzing Material Structures to Prevent Traumatic Brain Injuries. J Neurol Neurobiol 5(1): dx.doi.org/10.16966/2379-7150.154 


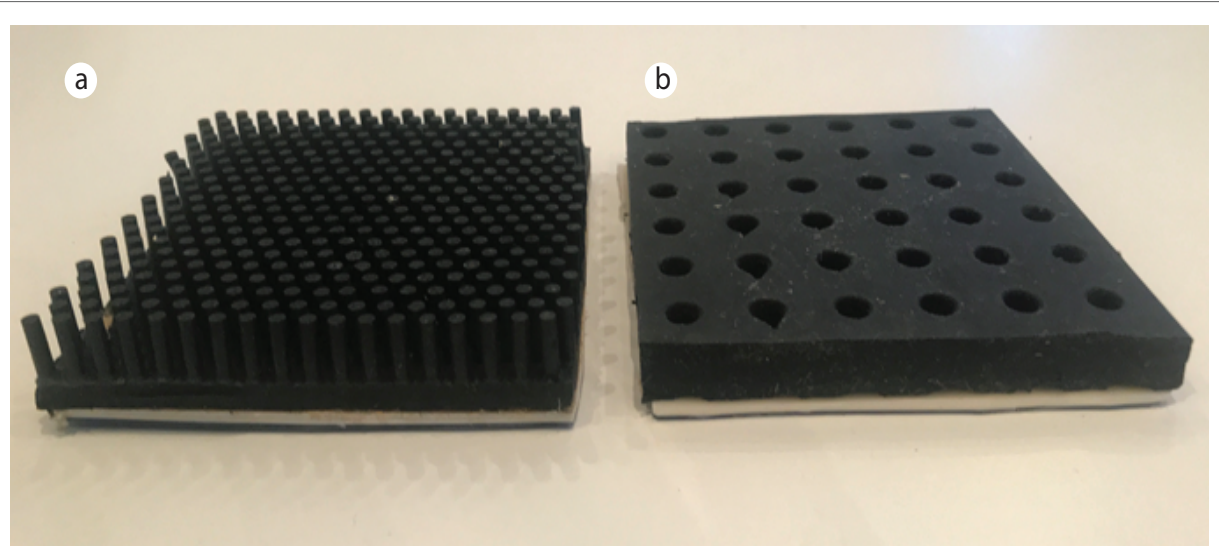

Figure 2: Showing the Sh 60 and Sh 81 with spikes (2a, left) and of Sh 50 and Sh 70 (2b, right).

Ref wooden board, $600 \mathrm{~mm}$.

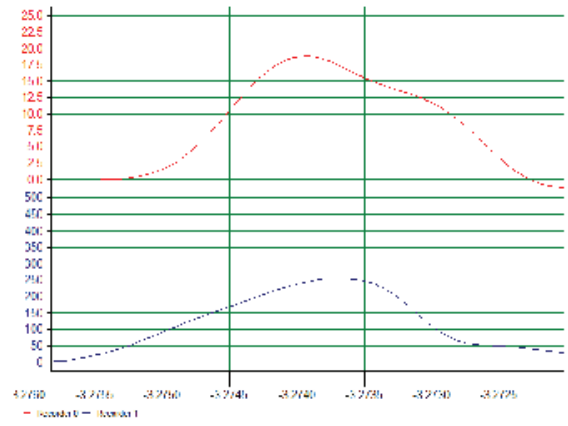

Force: $18.84 \mathrm{KN}$

Acceleration: $256.23 \mathrm{~g}$

Impact speed: $3.31 \mathrm{~m} / \mathrm{s}$
Sh $60,600 \mathrm{~mm}$.

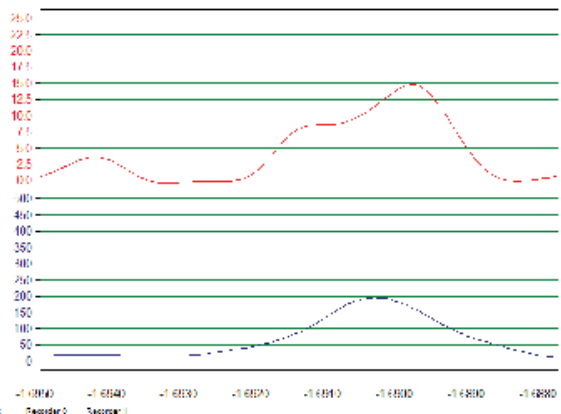

Force: $14.76 \mathrm{kN}$

Acceleration: $195.37 \mathrm{~g}$

Impact speed: $3.33 \mathrm{~m} / \mathrm{s}$
Sh $81,600 \mathrm{~mm}$.

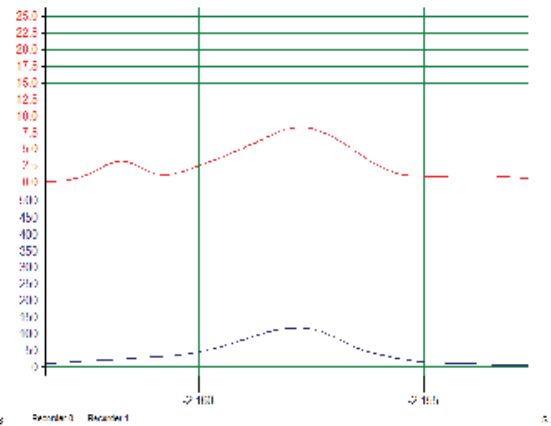

Force: $8.28 \mathrm{kN}$

Acceleration: $118.11 \mathrm{~g}$

Impact speed: $3.32 \mathrm{~m} / \mathrm{s}$

Figure 3: Results from the dynamic impact with steel body form on the polymers Sh 60 and Sh 81 and with their respective force, translational acceleration and impact speeds and compared to the reference wooden board.

Denatured electrophoresis: The ten force treated samples did not show any laminin fragmentation or aggregation or any significant differences compared to control samples (Figure 4). Also, in contrast to earlier force treated samples no major low-molecular weight fragments of laminin were observed. Further, by comparing the pattern between control and force treated samples there was no extra band found in the analysis. Thus, there seems to be no substantial difference compared to laminin controls between the two Sh 60 and Sh 81 polymers.

EM: Dynamic impact with steel head form did not cause any major visual change to laminin samples as compared to control specimen in negative stain EM (Figure 5). Increased aggregation of laminin, as was observed to take place in laminin samples with dynamic impact force without the polymer structures [4], was rare.

\section{Dynamic impact with Hybrid III head form}

In contrast to the steel body form having a pure steel surface, the Hybrid III head was equipped with a softer surface. From figure 6 it is shown that the dynamic impacts from six controls were defined to an average of $496 \mathrm{G}$ after approximately $0.0045 \mathrm{~s}$ (B). This was followed by some bruises of unknown origin (A). Then SH 70 showed a reduction of the translational acceleration to an average of $296 \mathrm{G}$ after approximately $0.005 \mathrm{~s}(\mathrm{C})$, thus reducing the acceleration with 40 $\%$. The reduction with Sh 50 was set to $238 \mathrm{G}$ after approximately 0.005 $\mathrm{s}(\mathrm{D})$ responding to $52 \%$ reduction. Also, the forces were reduced from initially $19,0 \mathrm{kN}$ to $11,13 \mathrm{kN}$ with Sh 70 and to $9,0 \mathrm{kN}$ with Sh 50 , thus a significant reduction.

Denatured electrophoresis: As was found in the electrophoresis following the steel body form impact, no strong fragmentation of laminin into new low-molecular weight bands or aggregation of the laminin structures were detected compared to controls. Also, no difference was observed between the different laminin bands in tested Sh 50 and Sh 70 polymer materials (Figure 7).

EM: When analysing the laminin with EM there seems to be no difference between the control samples and polymer structures. The use of polymer materials Sh 50 and Sh 70 did not show any difference in the laminin structures between the two tested polymers. Nor was major aggregation detected on the laminin samples (Figure 8). 


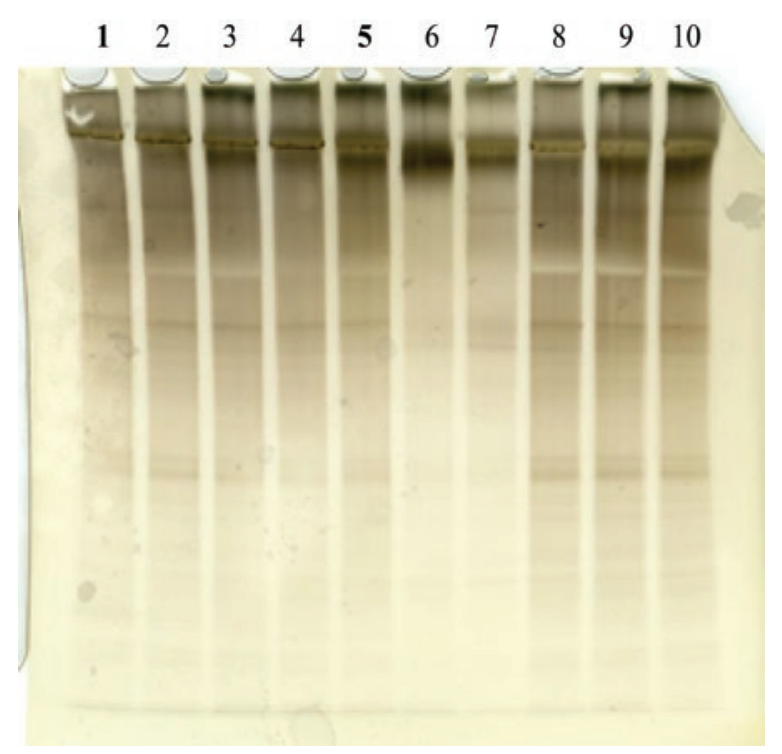

Figure 4: The different laminin bands in all of the ten polymers tested with the steel body form Lanes 1 and 5 are those two showing the electrophoresis response following the mechanical dynamic impact presented above.

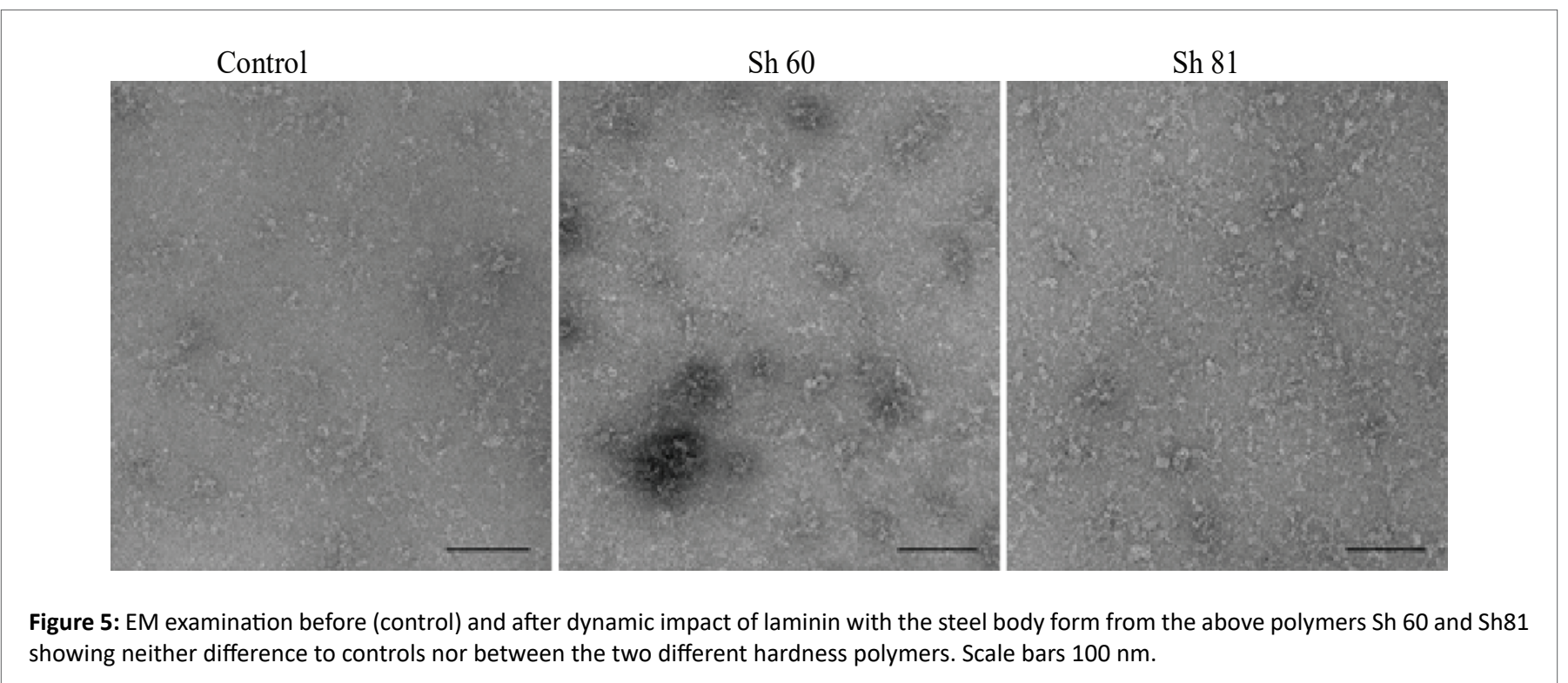

\section{Discussion}

The present results show for the first time that analyzing proteins with electrophoresis and EM in the search for improved protection material against traumatic brain injury may be an important complement to existing simulating and mechanical evaluations. The results from both steel body (Figure 3) as well as Hybrid III (Figure 6) impacts show that the present polymer materials have a positive outcome when it comes to save the laminin structures for a normal cellular metabolism following an impact. Also, the difference in hardening, design and types of spikes of the material is of importance to reduce the absorption of the impact force to the head. Of special interest is the figure 6 showing a clear picture of polymer materials compared to controls. However, based on the present results one must bear in mind that it is not possible to exactly quantify whether or not there are any denaturations of laminin structures. Comparing the mechanical results shown in figure 3 and figure 6, it is clear that the impact force reduced regardless of impact loading. Also, it is plausible that the softer polymers tested herein may be used instead of today's existing hard outer shell in helmets. Further, by using laboratory methods shown in figure 3 and figure 6 it is relevant to differentiate between less preventative materials from more optimal materials. Thus, analyzing mechanical force and acceleration in combination with molecules active in cellular metabolism such as protein structures may become the ultimate choice in finding the most effective material to prevent traumatic brain injury and future dementia in all ages. Elderly people ageing 65+ have an increased risk of head injury mainly due to imbalance and fall. A Swedish investigation has recently 
shown that traumatic brain injury substantially increases the risk of developing dementia among elderly people [12]. To prevent geriatric dementia following traumatic brain injury it is important to find any basic denominators between traumatic brain injury and geriatric dementia. In simulation and laboratory studies we found a protein disturbance including fragmentation and aggregation after both dynamic and static impacts to the head [4]. The protein disturbance seems to be in line with the protein disturbances observed among elderly people with geriatric dementia. The protein disturbance may, among other clinical conditions, result in swollen brain tissue and which is devastating, especially among elderly people. The same holds true also among younger and adult athletes. Despite new and improved head protection systems the number of head injuries are still unacceptable high. By using evaluation on a molecular level as shown in the present study, the search for the most optimal head protection systems may be facilitated. In common for traumatic brain injury and geriatric dementia, no matter of age, is thus a metabolic disturbance in protein metabolism and which may, to some extent, be prevented by different innovative safety products. All these innovations aimed for adult and elderly people have the potential to absorb the transferred energy into the head and brain tissue following a fall. Thus, the most optimal activity to avoid injuries among elderly individuals and to some extent consider the reduction of dementia after traumatic brain injury is to fully prevent them and this opens up for a number of innovative safety products including construction of safety systems like headband, gloves and floors. Besides, increased physical exercise has become very intense among the population all ages as it may help to keep alert also in elderly persons of $65+$. As a consequence, there is a need for the construction of new safety products but also improvement of existing preventative products when it comes to protect especially the head with brain tissue against traumatic brain tissue impacts of different severities and its secondary complications such as cytotoxic brain tissue edema and dementia. Today most of the evaluations of safety products rely on mechanical impacts in the laboratory sphere. Indeed, new insight to the consequences of dynamic and static impact to the head and brain tissue has seen the light during the last decades. Among the laboratory investigations to be mentioned are algorithm methods which have become popular to use in the laboratories worldwide. Indeed, algorithms are procedures of well-defined instructions designed to improve the pure mechanical investigations. However, as always when using various models for calculations such as finite element methods, data are taken from the human data base and included in these methods. Further, these algorithms often require an end of the simulation procedure. This is in contrast to secondary complications in traumatic brain injuries which continue long after the onset of the impact to the head. Also, it must be stressed that such models are just mathematical models built on different assumptions and simplifications that cannot be implicated in advanced systems like the human brain and other body organs. Thus, there is a need for better and more advanced analyses taking into consideration the molecular aspect following an impact.

To further improve evaluations of new material and safety products, there is a need to focus on the interference of dynamic and static impact also on a molecular level as mentioned above. In this regard there are a high number of proteins distributed in the whole human brain and which should respond to a dynamic or static impact when severe enough. Thus, earlier simulation methods and laboratory investigations have shown on fragmentation and aggregation of proteins when the impact is severe enough [13-15]. Of special interest are the proteins laminin which are abundant in the human body and which have been used for laboratory investigations [3]. The laminins belong to a large family of at least 16 isoforms in higher

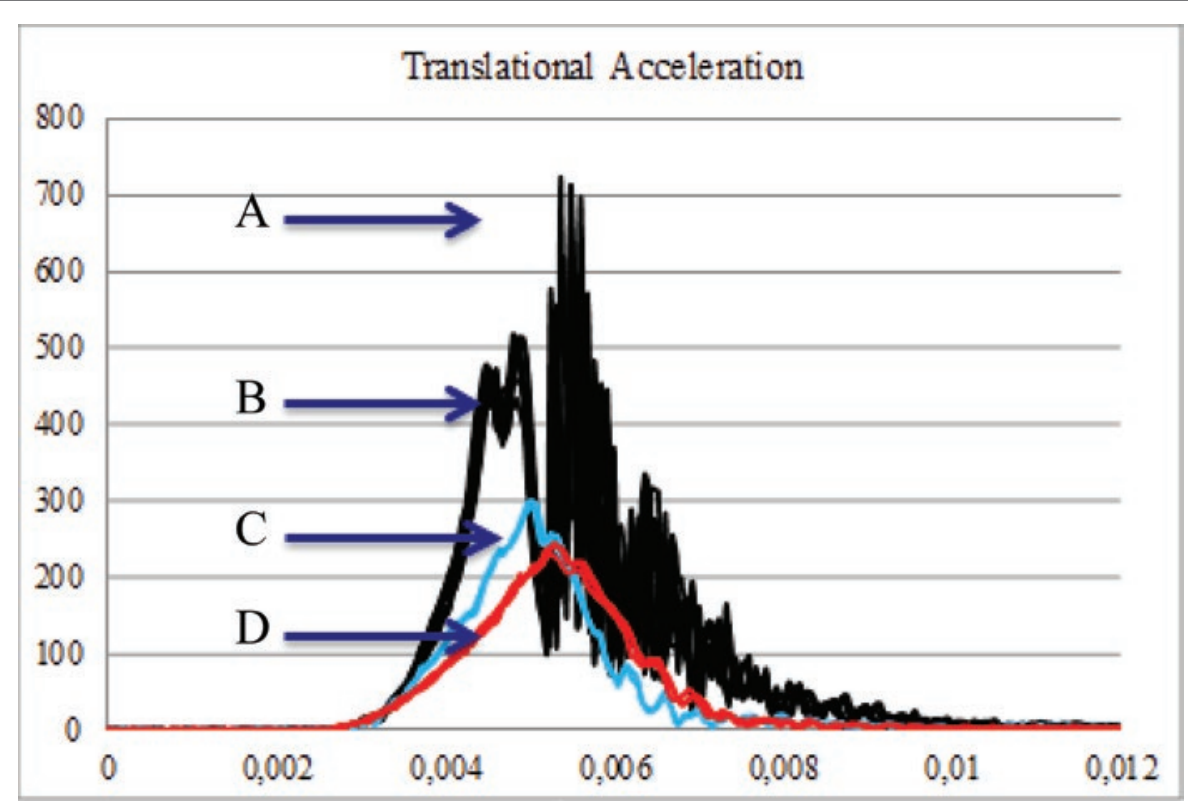

Force: $19,0 \mathrm{kN}$

B Acceleration: $496 \mathrm{G}$

Impact speed: $3.31 \mathrm{~m} / \mathrm{s}$
Force: $11,13 \mathrm{kN}$

C Acceleration: $296 \mathrm{G}$

Impact speed: $3.33 \mathrm{~m} / \mathrm{s}$
Force: $9,0 \mathrm{kN}$

D Acceleration: $238 \mathrm{G}$

Impact speed: $3.32 \mathrm{~m} / \mathrm{s}$

Figure 6: Translational acceleration (G) following dynamic impact with Hybrid III head form. A=bruise at impact, B=control from six impacts, $C=S h$ 70 from three impacts and $D=S h 50$ from three impacts. 


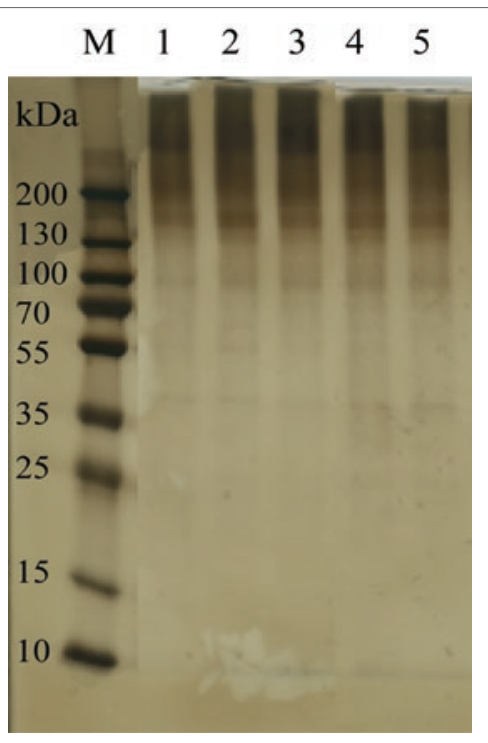

Figure 7: SDS page for laminin samples tested with the Hybrid III head. Lane $M$ is the molecular marker, where as the laminin samples from experiments with Sh50 polymers are shown on lanes 1-3 and two with Sh70 on lanes 4-5.
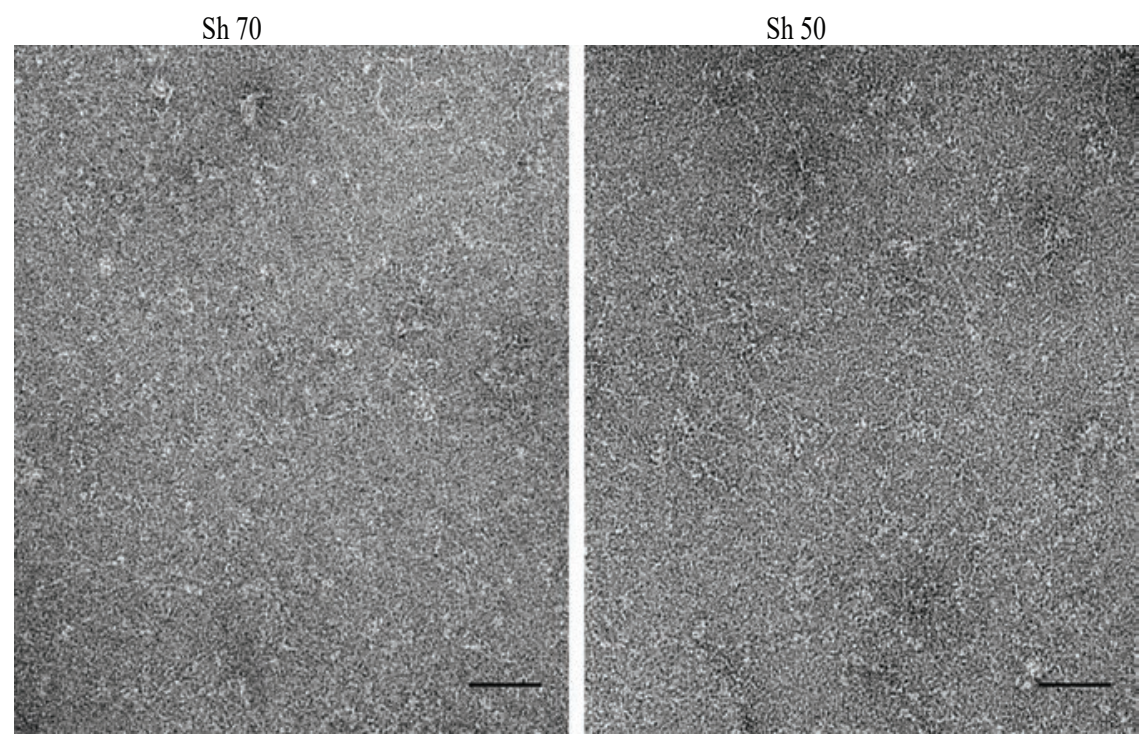

Figure 8: EM examination after dynamic impact of laminin with the Hybrid III head form with the polymers Sh 70 and Sh 50 . Scale bars $50 \mathrm{~nm}$.

organisms. The different activities of laminins are important for the basement membrane contributing to the structure of extracellular matrix thereby influencing the behavior of associated cells including adhesion, differentiation, migration, et cetera [3]. Thus, the potential use of laminins in clinical neuroscience focusing on the treatment of secondary complications may in the future become important when it comes to better understanding of the secondary complications such as cytotoxic brain injury edema and dementia after traumatic brain injury. In the present study two different impact dummies were used. The hard steel body form had a reference wooden material as basal platform and with the polymer products in between them. In contrast, the softer Hybrid III head form had a platform made by steel and with the polymer material products in between them. The conclusion is that the polymer products caused a reducing effect regardless of dummy construction or basal platform impacting the polymer and which may have implications when it comes to develop new analyzing methods at cellular levels or molecular levels in the future. In an earlier study we showed that laboratory dynamic impacts of around $500 \mathrm{G}$ resulted in fragmentation and aggregation to the laminin structure [3]. To the best of our knowledge the present study shows for the first time that polymer structures of different construction and different softness or hardness have the capacity to reduce both the force as well as the translational acceleration substantially in parallel with saving the mature laminin structure as confirmed with denatured electrophoresis and EM. These materials and others 
not investigated in this study may open a new insight of analyzing methodology by using laboratory technology with that of molecular substances like proteins.

\section{Conclusion}

Connecting algorithms for simulation and mechanical laboratory methods with that of analysis on a molecular level does not only have the potential to find the most optimal safety products when it comes to primary prevention of traumatic brain injuries and related dementia. It may also result into a better insight in understanding secondary complications such as cytotoxic brain tissue edema and dementia and the overall treatment of injuries in clinical neuroscience, no matter of the victims' age when an impact is received.

\section{Authors Contributions}

Hans von Holst designed the experiments and drafted the manuscript. $\mathrm{HvH}$ and DL were responsible for the dynamic laboratory tests, respectively. P.P. and H.H. analyzed the results of EM. R.B.K. analyzed the results of electrophoresis. All authors provided feedback and revised the manuscript.

\section{Competing Interest}

The authors declare no competing financial interests.

\section{References}

1. Pedersen K, Fahlstedt M, Jacobsson A, von Holst H (2015) A National Survey of Traumatic Brain Injuries Admitted to Hospitals in Sweden from 1987 to 2010. Neuroepidemiology 45: 20-27.

2. Swedish Civil Contingencies Agency, MSB (2014) Injuries among elderly people in Sweden during 2010-2011.

3. Aumailley M (2013) The Laminin Family. Cell Adh Migr 7: 48-55.

4. von Holst $\mathrm{H}$, Purhonen P, Lanner D, Kumar RB, Hebert H (2018) White Shark Protein Metabolism may be a Model to Improve the Outcome of Cytotoxic Brain Tissue Edema and Cognitive Deficiency after Traumatic Brain Injury and Stroke. J Neurol Neurobiol 4: 1-7.
5. Videby M, Andersson K-G (2014) A steel head form, MEP- platform (ASTM 1446), Research Institutes of Sweden.

6. Halldin P (2014) CEN/TC 158-WG11, Rotational test methods. ASTM New Orleans.

7. Integrated Pressure Sensor (2007) Freescale semiconductor. MPX5010. Rev 11. 01.

8. Shahbazi Z (2015) Mechanical model of hydrogen bonds in protein molecules. American J Mech Eng 3: 47-54.

9. Weber K, Osborn M (1969) The reliability of molecular weight determinations dodecyl sufate-polyacrylamidegel electrophoresis. J Biol Chem 244: 4406-4412.

10. Booth DS, Sakar-AA, Cheng $Y$ (2011) Visualizing proteins and macromolecular complexes by negative stain EM: from grid preparation to image acquisition. J Vis Exp 22.

11. De Carlo S, Harris JR (2011) Negative staining and cryo-negative staining of macromolecules and viruses for TEM. Micron 42: 117131.

12. Nordström A, Nordström P (2018) Traumatic brain injury and the risk of dementia diagnosis: A nationwide cohort study. PLoS Med 15: e1002496.

13. Holst Hv, Li X (2013) The dynamic triple peak impact factor in traumatic brain injury influences native protein structures in gray and white matter as measured with computational simulation. Neurol Res 35: 782-789.

14. von Holst $\mathrm{H}$, Li X (2013) Numerical impact simulation of gradually increased kinetic energy transfer has the potential to break up folded protein structures resulting in cytotoxic brain tissue edema. J Neurotrauma 30: 1192-1199.

15. von Holst $\mathrm{H}$, Li X (2015) Higher impact energy in traumatic brain injury interferes with noncovalent and covalent bonds resulting in cytotoxic brain tissue edema as measured with computational simulation. Acta Neurochir (Wien) 157: 639-648. 